\title{
Efecto del glicerol como fuente de carbono adicional en la obtención de biomasa y ácidos grasos en cultivos chlorella vulgaris en un foto-biorreactor de panel plano a escala de laboratorio
}

pags $8-20$

Grupo de Investigación: Biotecfua

Línea de investigación: Aprovechamiento de residuos agroindustriales

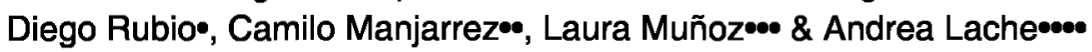

Recibido: 5 de junio de 2015

\section{RESUMEN}

En este proyecto de investigación se trabajó con la micro-alga Chlorella Vulgaris, con el objetivo de evaluar la influencia del glicerol, como nueva fuente posible de carbono, para la producción de biomasa y ácidos grasos. Se utilizó como medio de cultivo el fertilizante foliar (conocido comercialmente como FOLIAGRO) por poseer los macro y micronutrientes necesarios para el crecimiento adecuado de la micro-alga. Para la investigación, fueron evaluadas tres diferentes concentraciones de glicerol $(10,15$ y $20 \mathrm{~g} / \mathrm{L}$ ) en un foto-biorreactor de panel plano, con capacidad de trabajo de 1,4 L. El crecimiento microalgar se llevó a cabo en incubadoras, donde se monitorean las condiciones adecuadas de crecimiento. La agitación se realizó por medio de bombas para acuario con capacidad de $4 \mathrm{~L} / \mathrm{min}$, fotoperiodo de

\section{Aceptado: 12 de junio de 2015}

12L: 120 (Luz, Oscuridad), intensidad lumínica con lámparas halógenas de luz rojas y $8 \mathrm{~W}$, temperatura de $21^{\circ} \mathrm{C} \pm 3{ }^{\circ} \mathrm{C}$ y $\mathrm{pH} 6-7$; y el crecimiento se determinó por medio de conteos en cámara de Neubauer. Para la obtención de biomasa se realizaron los procesos secuenciales de sedimentación, centrifugación y liofilización. Después, fue evaluada la biomasa seca por medio de una cromatografía de gases, acoplada a su espectro, para determinar así el perfil lipídico de la misma. El glicerol demostró ser una fuente de carbono útil en el crecimiento de C. Vulgaris. La concentración de $15 \mathrm{~g} / \mathrm{L}$, resultó ser el medio más favorable para el crecimiento celular, durante los 12 dias de experimentación (crecimiento máximo $2,29 \times 10^{7} \mathrm{Cel} / \mathrm{mL}$ ).

Palabras clave: Chlorella Vulgaris, ácidos grasos, producción de biomasa, glicerol, biodiesel.

- Biólogo U. Militar, PhD. biotecnología U. Nacional. diego.rubio@investigadores.uamerica.edu.co

-. Estudiante Ingeniería Química Fundación Universidad de América.

... Estudiante Ingenieria Química Fundación Universidad de América.

.... Ing. Química Fundación Universidad de América MSc. Energías alternativas U. Menéndez Pelayo.

andrea.lache@investigadores.uamerica.edu.co 
LÍNEA DE INVESTIGACIÓN: APROVECHAMIENTO DE RESIDUOS AGROINDUSTRIALES

\section{ABSTRACT}

In this research project we worked with the microalga Chlorella Vulgaris, in order to evaluate the influence of glycerol as a new possible source of carbon for the production of biomass and fatty acids. It was used as a cultivate medium (commercially known as FOLIAGRO) like a foliar fertilizer, by having the macro and micro nutrients, needed for the proper growth of the micro-alga. For the research were evaluated three different concentrations of glycerol $(10,15$ and $20 \mathrm{~g} / \mathrm{l})$ in a photo-bioreactor with a flat panel, and a working capacity of 1,4 L. Micro-algar growth was held in incubators, monitored with appropriate conditions of growth. The agitation was conducted by aquarium pumps, with a capacity of $4 \mathrm{~L} / \mathrm{min}$, photoperiod of 12 L: 12th (light: dark), light intensity red light halogen lamps with $8 \mathrm{~W}$, temperature of $21^{\circ} \mathrm{C} \pm 3^{\circ} \mathrm{C}$ and $\mathrm{pH}$ 6-7; the growth was determined by counts in Neubauer Chamber. For obtaining of biomass were sequential processes of sedimentation, centrifugation and lyophilization. It was evaluated after the dry biomass with a gas chromatography, coupled to mass spectrum and thus determine the lipid profile. Glycerol proved to be a source of useful carbon in the growth of C. Vulgaris. $15 \mathrm{~g} / \mathrm{l}$ concentration turned out to be more favorable for cell growth medium during the 12 days of experimentation (maximum growth 29 $\times 107 \mathrm{Cel} / \mathrm{mL}$ ).

Key Words: Chlorella Vulgaris, fatty acids, biomass production, glycerol, biodiesel.

\section{INTRODUCCIÓN}

El constante crecimiento en la demanda de lípidos y ácidos grasos para uso industrial, ha generado la necesidad de encontrar nuevas y mejores fuentes para la obtención de los mismos. Por eso se buscan mecanismos que trabajen de forma eficiente, eliminando los daños ambientales y, a su vez, permitiendo abastecer satisfactoriamente la demanda de estas materias primas. Se considera que los combustibles provenientes de biomasa microalgal tienen un alto potencial y pueden verse como una de las alternativas mas promisorias para el reemplazo del petróleo en el mediano plazo dado su desempeño en motores diésel y la disminución de liberación de $\mathrm{CO}_{2}$ (Demirbas y Demirbas, 2011).
Las microalgas son un grupo diverso de microorganismos procarióticos y eucarióticos fotosintéticos, que crecen rápidamente debido a su estructura simple. Potencialmente pueden ser empleadas para la producción de biocombustibles (biodiesel, biogás y bio-hidrógeno) ambientalmente sostenibles y económicamente eficaces (Torres et al., 2013).

La obtención de ácidos grasos y lípidos, a partir de biomasa micro-algar, es un tema que se viene estudiando desde hace mucho tiempo, por ser una nueva alternativa para producir energías más limpias y amigables con el medio ambiente, y reduciendo costos a la hora de su producción. En comparación con otras materias primas, no son fuente de alimento humano, no presentan gran complejidad química en su estructura, lo que hace su manipulación y modificación más sencilla. El cultivo de las micro-algas y sus condiciones, permite que se produzcan a gran escala y en muy cortos periodos de tiempo. Además, por ser fotosintéticas, absorben en gran medida el dióxido de carbono presente en la atmosfera. Es por esto que el grupo de Biotecnología de la Universidad América (BIOTECFUA) centra sus esfuerzos en evaluar las mejores condiciones de cultivo, los mejores medios químicos y físicos para la producción de lípidos y ácidos grasos micro-algares, así como los mejores métodos para la extracción, clasificación y cuantificación de los mismos.

La obtención de biomasa microalgal y su viabilidad desde el punto de vista industrial requiere conocimiento sobre los factores que permitan disminuir los costos de producción y aumentar la cantidad de biomasa obtenida; dentro de los factores que aun requieren evaluación se encuentran sustancias que puedan ser empleadas como fuentes de Carbono y Nitrógeno y no representen costos excesivos a los procesos de producción (Deschenes et al., 2015).

Este estudio se centrará en evaular el glicerol como fuente de carbono adicional en el medio de cultivo para la producción de biomasa y lípidos a partir de microalgas. Esto con el fin de 
aumentar la obtención de biomasa y ácidos grasos, para hacer viable la producción de biocombustibles a partir de microalgas, lo que es de gran interés en el desarrollo de bio-procesos sostenibles, para ser acoplados e implementados en la Ingeniería Química.

\section{MATERIALES Y MÉTODOS}

\section{Cepa micro-algar, condiciones de cultivo y medio de cultivo}

La cepa de trabajo es la micro-alga Chlorella Vulgaris, seleccionada por su alta concentración de clorofila y su alta capacidad de fotosíntesis. Tiene un contenido de lípidos de 5-58\% (porcentaje de biomasa peso seco) y una productividad lipidica de $11,2-40,0 \mathrm{mg} / \mathrm{L} /$ día.

El crecimiento micro-algar se llevó a cabo en incubadoras, donde se monitorean las condiciones adecuadas de crecimiento. La agitación se realizó por medio de bombas para acuario JAD con capacidad de $4 \mathrm{~L} / \mathrm{min}$, fotoperiodo de $12 \mathrm{~L}$ : 120 (Luz, Oscuridad), intensidad lumínica con lámparas halógenas de luz rojas y $8 \mathrm{~W}$, temperatura de $21^{\circ} \mathrm{C} \pm 3^{\circ} \mathrm{C}$ y pH 6-7 (Li et al., 2007).

Se utiliza como medio de cultivo el fertilizante agrícola foliar, que posee nutrientes similares a los del medio Bold Basal. La concentración recomendada de medio de cultivo, preparado con foliar líquido llamado FOLIAGRO, es de $0,2 \%$ Volumen/Volumen.

\section{Crecimiento inicial de biomasa}

Se preparó el medio de cultivo a una concentración $0,2 \%$ VN de fertilizante con agua destilada. Para ello se utilizaron recipientes de vidrio con tapa y capacidad de $1 \mathrm{~L}$. Después de preparado, el medio de cultivo se esterilizó en una autoclave Tuttnauer.

Inicialmente la micro-alga fue puesta en crecimiento, en un medio de cultivo por un periodo de 12 días. Se inocularon $100 \mathrm{~mL}$ de micro-alga, por un litro de medio de cultivo en recipientes de vidrio con capacidad de $4 \mathrm{~L}$ cada uno, manteniendo una relación de 1:10 volumen/volumen. Fue dejado el sobrante de micro-alga como réplica. La relación inicial micro-alga-medio de cultivo, se estableció teniendo en cuenta que, durante la etapa de crecimiento, es importante que los microorganismos tengan buena disponibilidad de nutrientes minerales (Xu et al., 2006).

\section{Selección de concentración de glicerol a evaluar}

Las concentraciones de glicerol establecidas como parámetros experimentales, fueron seleccionadas con base a criterios teóricos (Estevez-Landazabal et al., 2013) soportados con un proceso previo de pre-experimentación. En estudios realizados anteriormente (Estevez-Landazabal et al., 2013) (Pestana y Pestana., 2012), se trabajó con concentraciones de glicerol $(1,5$ y $10 \%$ VN o g/L) (Kong et al, 2013). Para esta investigación se agregan dos nuevas concentraciones que no han sido reportadas previamente en literatura (15 y $20 \mathrm{~g} / \mathrm{L}$ ). Se realizó un pre-experimento con las 5 concentraciones seleccionadas, el cual consistió en evaluar el crecimiento celular de cada una, en un periodo de 5 dias. El glicerol con el que se decide trabajar, es glicerol grado USP de marca Carlo Erba.

\section{Crecimiento micro-algar en las concentraciones seleccionadas en el foto-biorreactor de panel plano}

Con base en los resultados de número de células por mililitro obtenidas en la fase de preexperimentación se seleccionaron las tres concentraciones de glicerol, que presentaron mayor crecimiento celular durante el pre-experimento $(10 \%, 15 \%$ y $20 \% \mathrm{~g} / \mathrm{L})$.

Una vez seleccionadas las concentraciones de glicerol que mayor cantidad de células por mililitro presentaron se realizó una nueva etapa de crecimiento micro-algar, en los foto-biorreactores de panel plano, con las concentraciones seleccionadas. 
Para los cultivos en Fotobiorreactor se utilizaron 3 fotobiorreactores tipo panel plano, cada uno con un volumen de trabajo de 1,4 L.La evaluación de las concentraciones finales seleccionadas se llevó a cabo en los fotobiorreactores.

Con el objeto de obtener una mayor concentración de biomasa mínima para las pruebas de laboratorio se utilizaron 3 recipientes adicionales de $4 \mathrm{~L}$ en los cuales se implementaron las mismas condiciones de cultivo de los fotobiorreac- tores. En cada recipiente se inocularon $300 \mathrm{~mL}$ de micro-alga, y $3 \mathrm{~L}$ de medio de cultivo con la concentración de glicerol respectiva.

Fueron conectadas dos mangueras lisas al recipiente: una para su aireación y otra para su desgasificación. Posteriormente los 3 fotobiorreactores y 9 recipientes de vidrio, fueron llevados a la incubadora por un periodo de 12 días. En la Figura 1 se muestra el montaje del experimento.

Figura 1. Fotobiorreactor de panel plano y sistema de cultivo en incubadoras
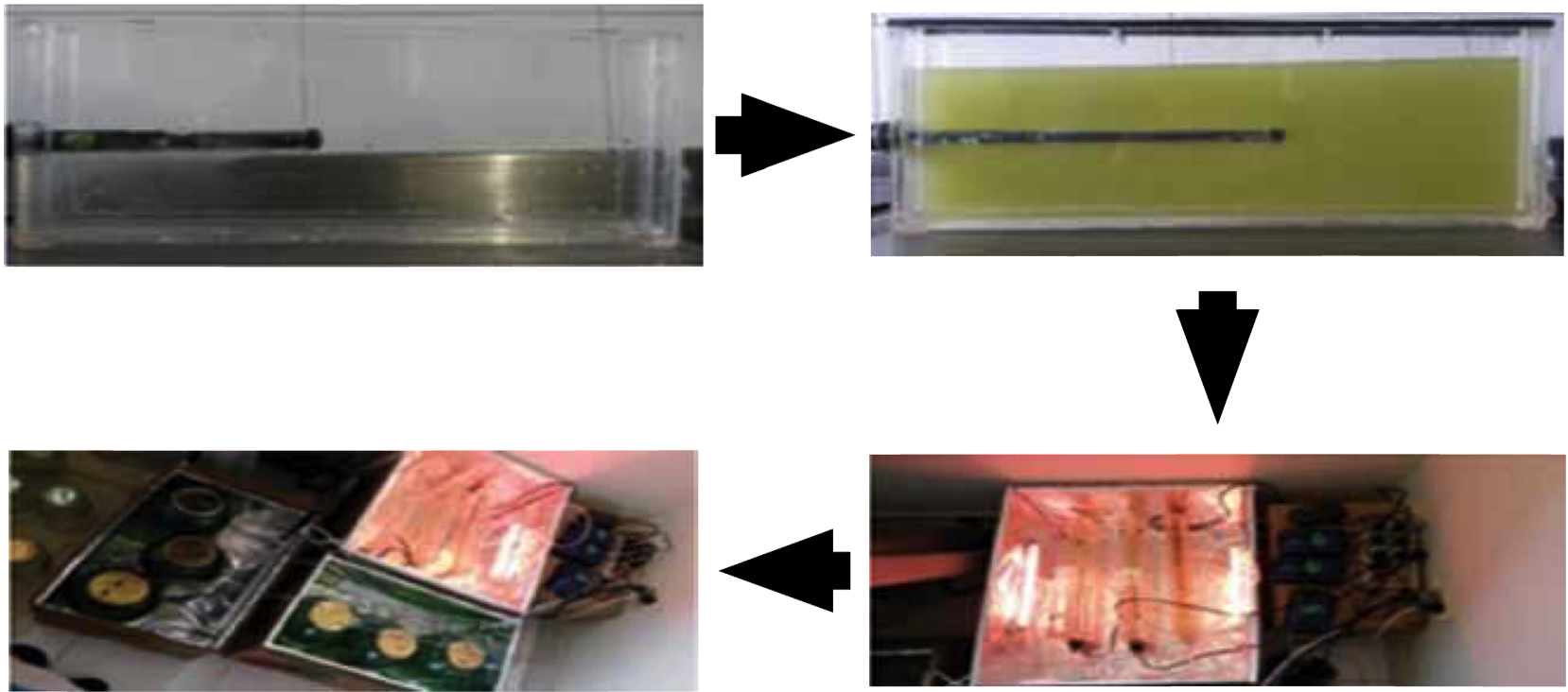

Fuente: Los Autores.

\section{Tratamiento de la biomasa}

Pasados los 12 días de crecimiento microalgar, se deja sedimentar la biomasa durante 48 horas por gravedad, y posteriormente el sobrenadante es retirado. Para la centrifugación, se usó una centrífuga con una velocidad angular de 4000 RPM por un periodo de 10 minutos. EI proceso de liofilización se llevó a cabo en el Laboratorio de Nutrición Animal de la Universidad Nacional de Colombia, sede Bogotá. La muestra centrifugada fue congelada a una temperatura de $-80^{\circ} \mathrm{C}$ durante 24 horas, y posteriormente fue liofilizada. Es decir, se retiró la humedad presente por sublimación (paso del estado sólido a vapor sin pasar por la fase líquida).

\section{Extracción de lípidos y perfil de ácidos grasos}

La extracción de los ácidos grasos se efectúa por medio del método Blight \& Dyer (Blight y Dyer, 1959), en donde la muestra se extrae con una solución metanol- cloroformo, en una proporción 2:1. La identificación y determinación de la composición de los ácidos grasos, se lleva a cabo por cromatografía de gases, empleando un cromatógrafo de columna capilar (GC - 14A). 


\section{RESULTADOS Y DISCUSIÓN}

\section{Crecimiento inicial de biomasa micro-algar}

La verificación del crecimiento del cultivo y la producción de biomasa se realizaron cualitativamente por cambios físicos como el color y el volumen del cultivo; así mismo se realizó seguimiento cuantitativo, a través de los conteos hechos por cámara de Neubauer, donde se comprobó el aumento diario de células en las muestras.

Los datos obtenidos sugieren que los cultivos microalgales tuvieron fase de crecimiento y exponencial (Madigan et al.,2000) sin embargo no se aprecia claramente la fase estacionaria lo que indica que posiblemente se podría obtener aun mayor concentración celular.

\section{Pre-experimento en concentraciones de glicerol determinadas}

Para el pre-experimento se utilizaron 6 recipientes de vidrio de $1 \mathrm{~L}$ en los que se inocularon $40 \mathrm{ml}$ de micro-alga y $400 \mathrm{~mL}$ de medio de cultivo con las concentraciones de glicerol respectivas: $1 \%, 5 \%, 10 \%, 15 \%$ y $20 \%$ de glicerol. La etapa de pre-experimentación para cada una de las concentraciones se llevó a cabo en 5 días (120 horas).

En la Gráfica 2, se observa que las concentraciones, generando mayor crecimiento microalgar y mejor rendimiento en la producción de biomasa, son las de $15 \%, 10 \%$ y $20 \%$, respectivamente. Se ve cómo la micro-alga se adapta más rápidamente a los medios de cultivo, con mayor porcentaje de glicerol, pues les ofrece un medio enriquecido con esta fuente de carbono.

La fase de adaptación al medio de cultivo para estas tres concentraciones, se lleva a cabo en 2 dias (24 horas). A partir de entonces se da inicio a la fase de crecimiento exponencial. La concentración de $20 \%$ de glicerol reporta un crecimiento máximo de $4,61 \times 10^{6} \mathrm{Cel} / \mathrm{mL}$, un valor menor al reportado por las concentraciones de $10 \%$ $\left(5,11 \times 10^{6} \mathrm{Cel} / \mathrm{mL}\right)$ y $15 \%\left(5,70 \times 10^{6} \mathrm{Cel} / \mathrm{mL}\right)$. Esto permite inferir que la concentración de $20 \%$ de glicerol en el medio de cultivo, comienza a saturar la misma de fuente de carbono, provocando inhibición en el crecimiento micro-algar.

Crecimiento micro-algar de las concentraciones seleccionadas en el fotobiorreactor de panel plano.

En la Grafica 3, se ve claramente la cinetica de crecimiento de cada concentración con respecto a las otras. El blanco es la muestra que más tiempo utiliza para adaptarse al medio de cultivo y la que menos crecimiento micro-algar demuestra. Para la concentración de $20 \%$ se ve una disminución significativa con respecto al crecimiento celular, de las concentraciones de $10 \%$ y $15 \%$, lo que permite inferir efectivamente, que la alta concentración de glicerina inhibe el crecimiento, pues satura de fuente de carbono el medio de cultivo.

Por otro lado, las concentraciones de $10 \%$ y $15 \%$, presentan un crecimiento celular constante. La cinetica de crecimiento micro-algar, en medio de cultivo con $15 \%$ de glicerol como fuente de carbono, es la más influyente en el experimento. Genera mayor concentración celular $\left(2,35 \times 10^{7}\right.$ Cel/mL) y menor tiempo de adaptación al medio de cultivo. Por lo anterior, fue la concentración seleccionada para pasar al proceso de obtención y tratamiento de la biomasa micro-algar.

La hipótesis nula (Ho) plantea que la concentración inicial micro-algar es la misma, tanto entre réplicas como entre experimentos. La hipótesis alternativa (H1), plantea que al menos uno de los experimentos o réplicas difiere de los demás.

Se realizó un análisis de varianza para los datos de esta gráfica, donde $\mathrm{F}$ es menor que el valor crítico para $F$, para una significancia de 0,05 . Esto comprueba que no existen diferencias significativas entre las concentraciones iniciales de cada experimento. 
LÍNEA DE INVESTIGACIÓN: APROVECHAMIENTO DE RESIDUOS AGROINDUSTRIALES

\section{Grafica 1. Curva de crecimiento de Chlorella vulgaris en medio foliagro}

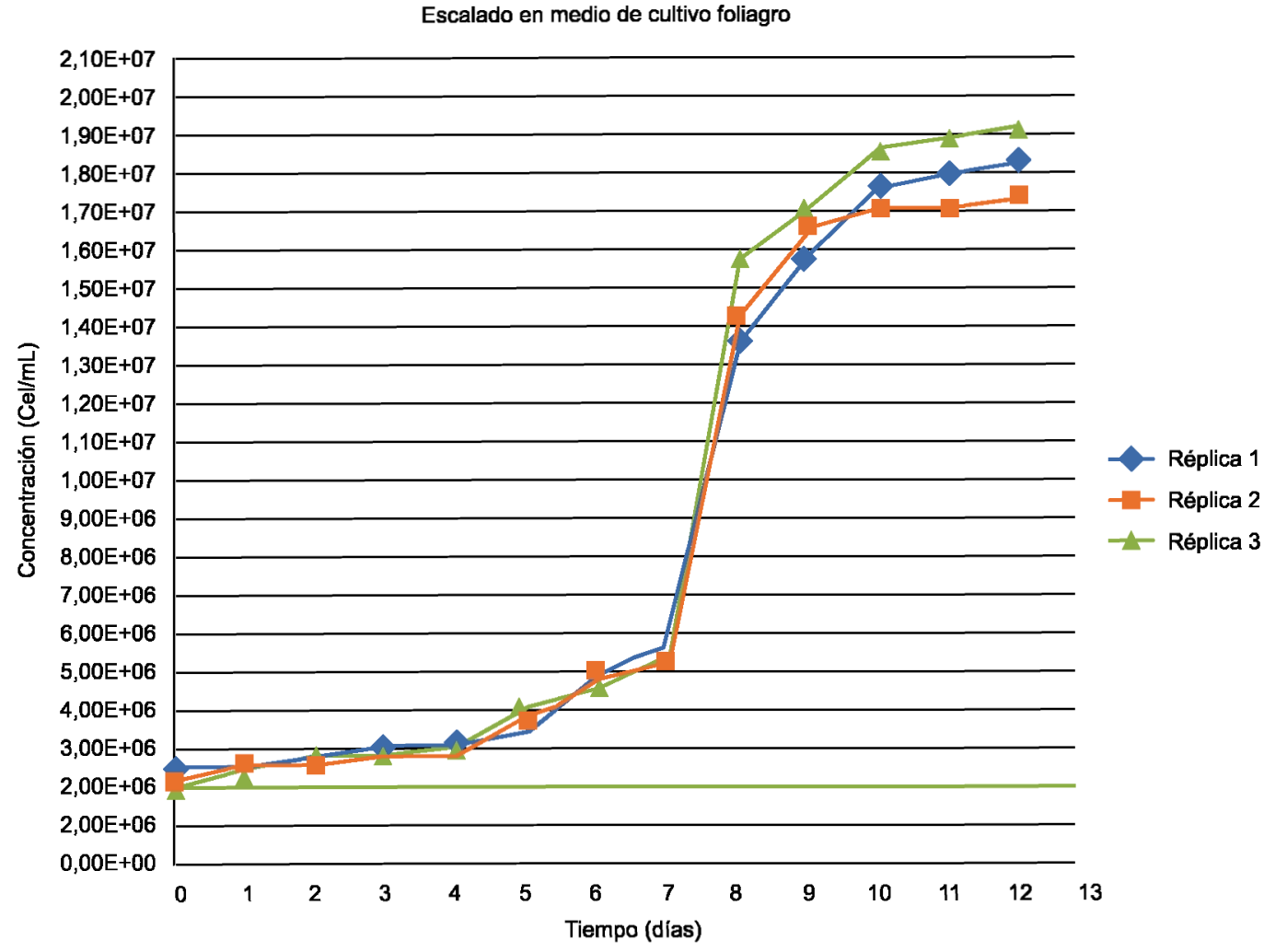

Fuente: Los Autores.

Gráfica 2. Curva de crecimiento promedio del pre-experimento.

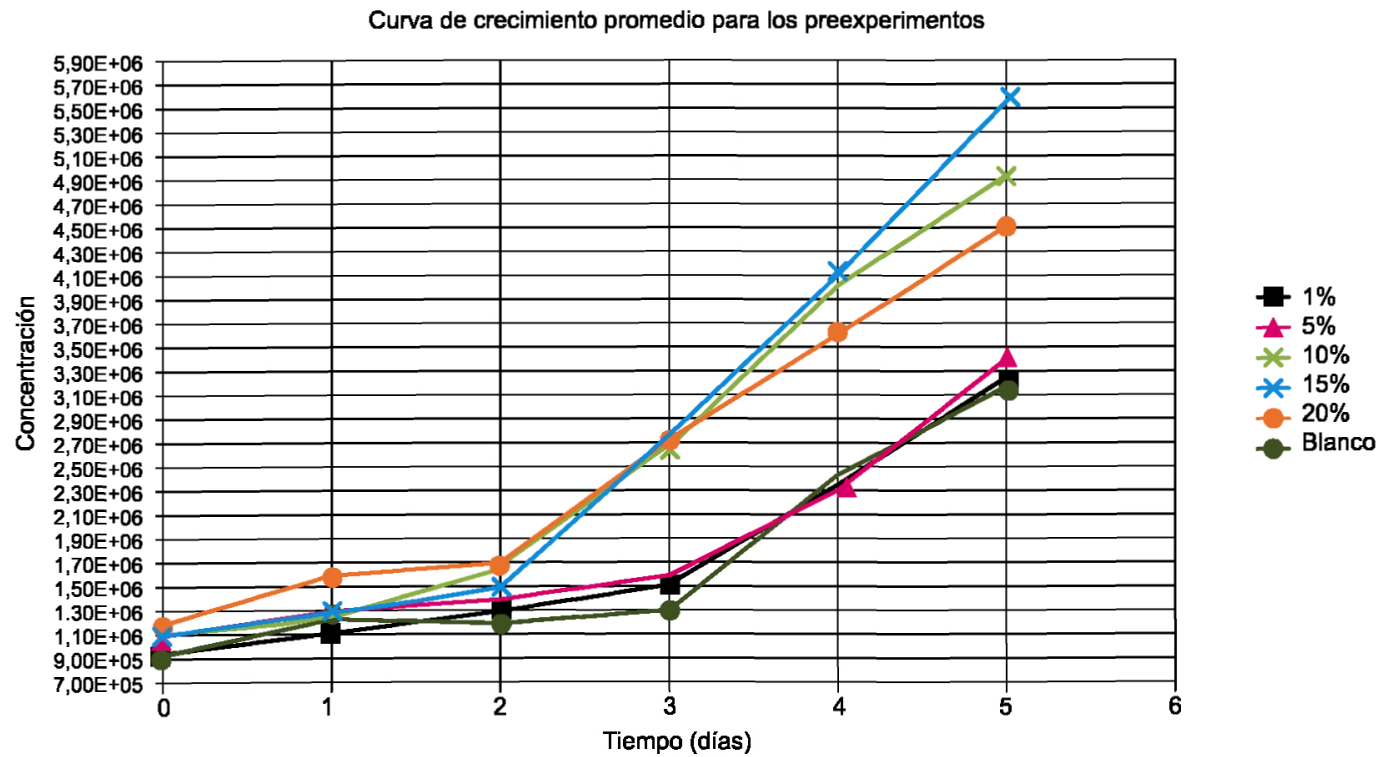

Fuente: Los Autores. 


\section{Gráfica 3. curva de crecimiento promedio del Experimento}

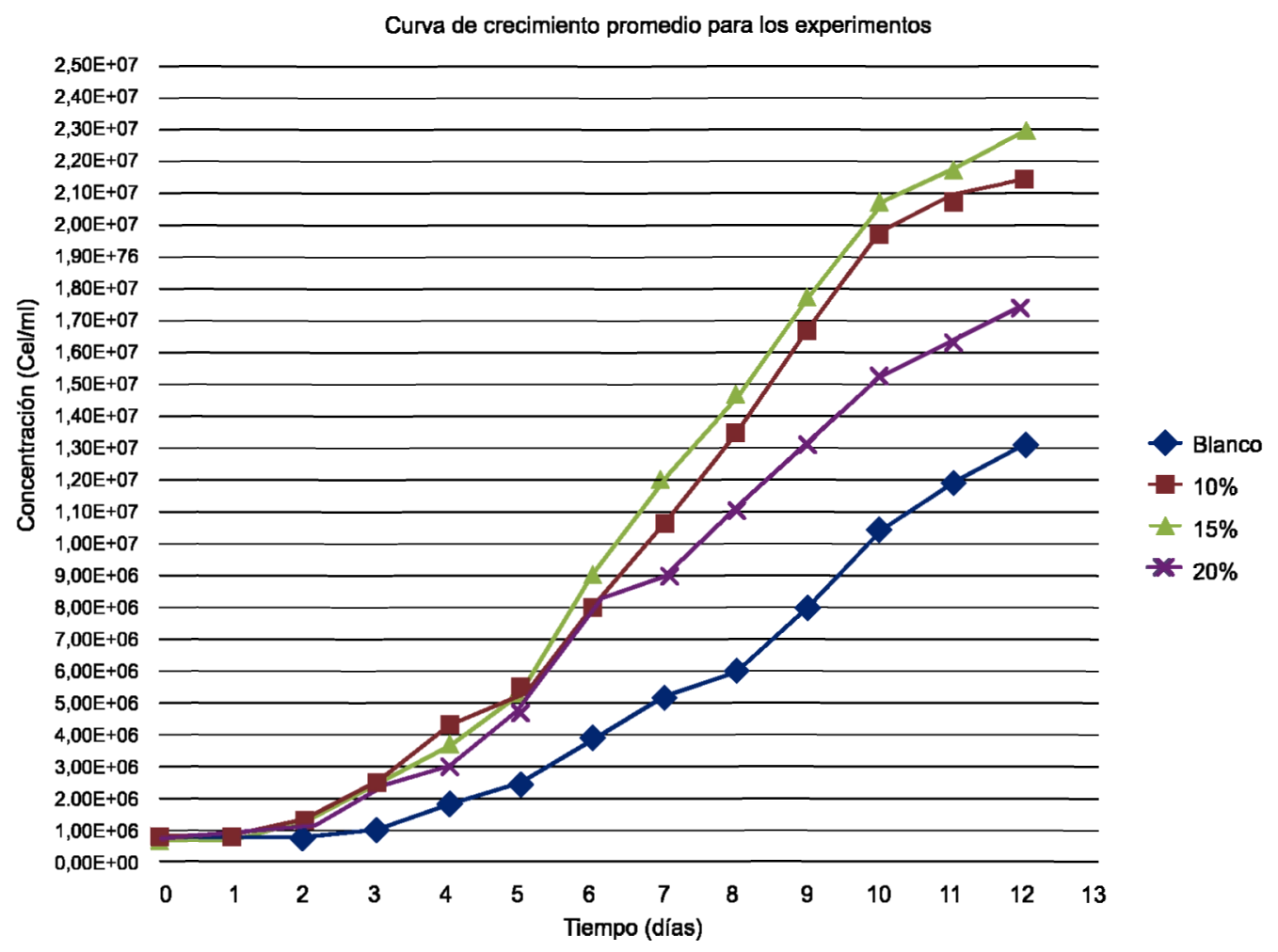

Fuente: Los Autores.

\section{Tratamiento de biomasa micro-algar}

Siguiendo el protocolo de tratamiento de biomasa micro-algar, descrito en la metodología, se deja sedimentar la micro-alga, encontrada en medio de cultivo con $15 \%$ de glicerol durante 48 horas; después se extrae el sobrenadante. Finalmente de $10020 \mathrm{~mL}$ de medio de cultivo con $15 \%$ de glicerol, se obtuvo $1680 \mathrm{~mL}$ de biomasa sedimentada, y se lleva al proceso de centrifugación.

La productividad varía con las diferentes concentraciones de Carbono estudiadas. Al aumentar la concentración de Carbono, el rendimiento se eleva en un $19,4 \%$, con lo cual se demuestra que una concentración de $15 \%$ aumenta la producción de biomasa en la microalga Chlorella vulgaris, en comparación con el medio de cultivo control, y a resultados reporta- dos por estudios referentes a la producción de biomasa micro-algar.

\section{Composición y perfil de ácidos grasos}

La composición de los ácidos grasos obtenidos (gráfica 4), está definida por un alto contenido de ácidos grasos poliinsaturados (PUFAs). Equivalen al 46,1\%, especialmente ácidos linoléicos y ácidos $\alpha$-linoléicos utilizados en la industria de alimentos. Un $29.5 \%$ corresponde a ácidos saturados, principalmente ácido palmítico y ácido esteárico, utilizados industrialmente para producir jabones, cosméticos, detergentes y lubricantes. Y un $24,4 \%$ de ácidos grasos mono-insaturados (MUFAs), como el ácido palmitoléico y ácido oleico, empleados en la fabricación de cosméticos, jabones, en la limpieza de metales y en la industria textil. 
LÍNEA DE INVESTIGACIÓN: APROVECHAMIENTO DE RESIDUOS AGROINDUSTRIALES

Tabla 1. Análisis de varianza entre las concentraciones iniciales de la tráfica

\begin{tabular}{|l|c|c|c|c|c|c|}
\hline $\begin{array}{c}\text { Análisis de } \\
\text { varianza } \\
\text { Origen } \\
\text { de las } \\
\text { variaciones }\end{array}$ & $\begin{array}{c}\text { Suma de } \\
\text { cuadrados }\end{array}$ & $\begin{array}{c}\text { Grados de } \\
\text { libertad }\end{array}$ & $\begin{array}{c}\text { Promedio } \\
\text { de los } \\
\text { cuadrados }\end{array}$ & F & Probabilidad & $\begin{array}{c}\text { Valor crítico } \\
\text { para F }\end{array}$ \\
\hline Entre grupos & $2,31588 \mathrm{E}+12$ & 2 & $1,15794 \mathrm{E}+12$ & 0,029812216 & 0,970651721 & 3,259446306 \\
\hline $\begin{array}{l}\text { Dentro de los } \\
\text { grupos }\end{array}$ & $1,39828 \mathrm{E}+15$ & 36 & $3,88412 \mathrm{E}+13$ & & & \\
\hline Total & $1,4006+15$ & 38 & & & & \\
\hline
\end{tabular}

Fuente: Los Autores.

Cuadro 1. Tratamiento de biomasa.

\begin{tabular}{|l|c|}
\hline Volumen de microalga total & $9 \mathrm{~L}$ \\
\hline Volumen sedimentado y centrifugado & $1680 \mathrm{~mL}$ \\
\hline Peso recipiente vacío & $7,1 \mathrm{~g}$ \\
\hline Peso recipiente + biomasa húmeda & $35,7 \mathrm{~g}$ \\
\hline Peso biomasa húmeda & $28,6 \mathrm{~g}$ \\
\hline Peso biomasa liofilizada (seca) & $1,75 \mathrm{~g}$ \\
\hline
\end{tabular}

Fuente: Los Autores.

Rendimiento y productividad volumétrica del FBR.

Cuadro 2. Resultados del rendimiento y productividad.

\begin{tabular}{|l|c|c|c|}
\hline & Rendimiento (g/L) & Productividad (g/d*L) \\
\hline Biomasa seca (g) & 1,75 & 0,194 & 0,016 \\
\hline Días & 12 & 0 & \\
\hline Volumen (L) & 9 & & \\
\hline
\end{tabular}

Fuente: Los Autores.

Debido a su alto contenido en (PUFAs), especialmente de omega 3 y omega 6 , esta biomasa es apta para el consumo humano. No es viable utilizarla para la obtención de biodiesel debido a que, por su alto contenido de ácidos grasos insaturados, la estabilidad oxidativa disminuye, así como también disminuye el calor de combustión, el índice de cetano del biodiesel, el punto de nube, la viscosidad cinemática y la lubricidad.

El desarrollo actual de la tecnología para la producción de biodiesel, a partir de micro- algas, tal como lo han estudiado (Bahadar \& Bilal Khan, 2013), es altamente susceptible a las condiciones de cultivo, tratamiento y extracción de los lípidos. Es justamente la extracción, una de las etapas más relevantes para que el proceso posterior de esterificación, o de licuefacción hidro-térmica (para el caso de Chlorella Vulgaris), permitan obtener biodiesel dentro de un esquema sostenible, contemplando el balance de $\mathrm{CO}_{2}$ y energía, el impacto medio ambiental $\mathrm{y}$, no menos importante, los costos de producción (Slade \& Bauen, 2013). 


\section{Gráfica 4. Composición de ácidos grasos}

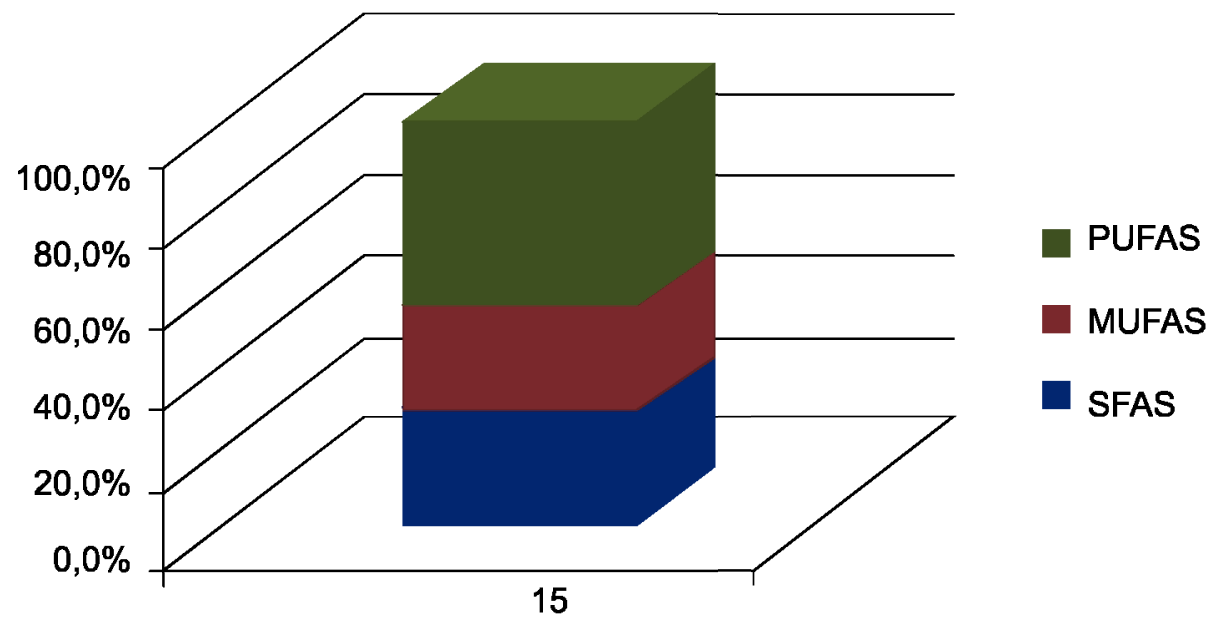

Fuente: Los Autores.

\section{Gráfica 5. Perfil de ácidos grasos}

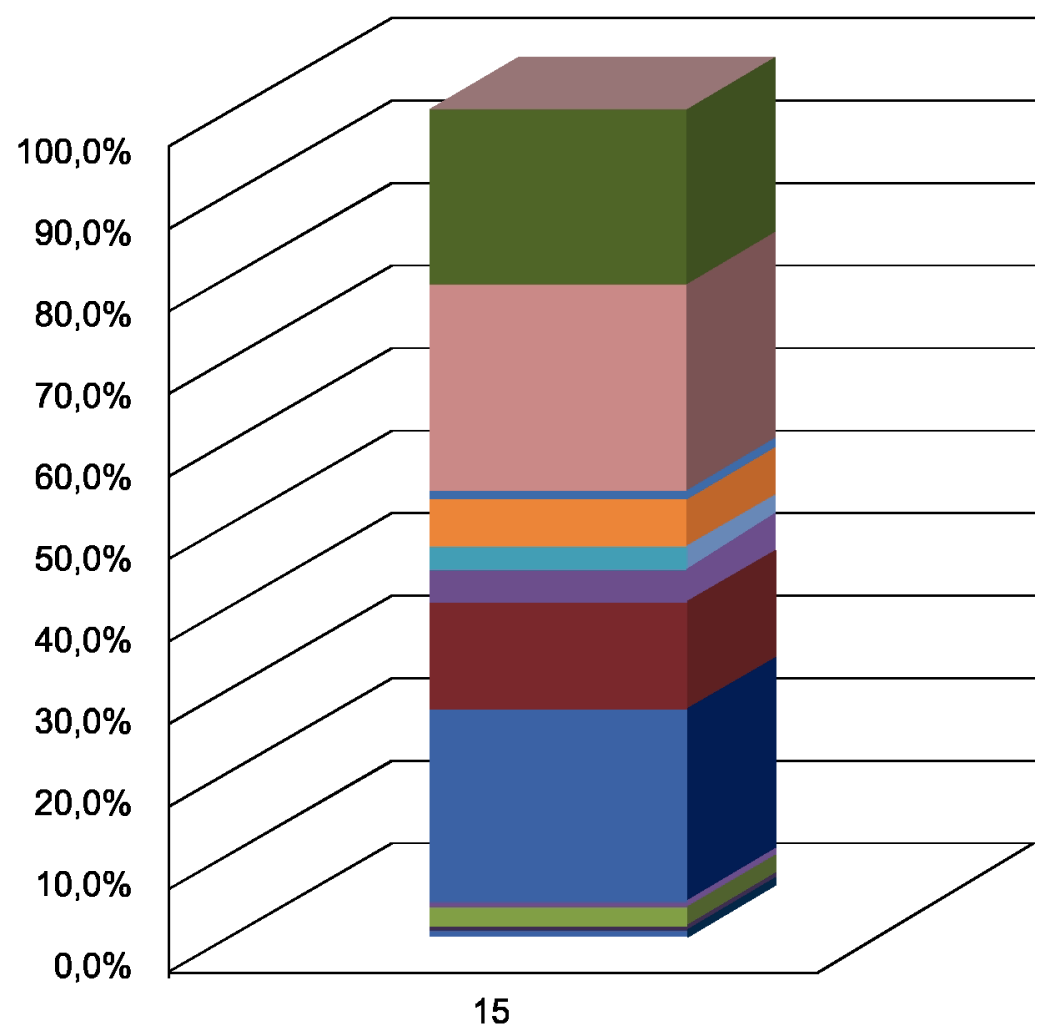
Ácido
docosahexaenoico
- Ácido behénico
Ácido
eicosapentenoico
- Ácido behénico
- Ácido araquídico
Ácido $\alpha$-linolenico
Ácido linoléico
Ácido vaccenico
Ácido oléico
- Ácido esteárico
Ácido
heptadecenóico
- Ácido margárico
- Ácido palmitoléico
- Ácido palmítico
Ácido
pentadecenóico
- Ácido
pentadecílico
- Ácido miristoléico
- Ácido mirístico
- Ácido cáprico
- Ácido caprílico

Fuente: Los Autores. 
LÍNEA DE INVESTIGACION: APROVECHAMIENTO DE RESIDUOS AGROINDUSTRIALES

\section{CONCLUSIÓN}

Las condiciones de cultivo seleccionadas: nutrientes (glicerol como fuente de carbono), temperatura $\left(21^{\circ} \mathrm{C}+3^{\circ} \mathrm{C}\right), \mathrm{pH}(6-7)$, agitación (bombas de acuario con capacidad de 4 Lmin), iluminación (lámparas halógenas de luz roja y $8 \mathrm{~W}$ ) y fotoperiodo (12 Luz: 12 Oscuridad), para el crecimiento y escalamiento de la micro-alga, demostraron ser las adecuadas para la acumulación y obtención de biomasa y ácidos grasos, debido a que por medios cuantitativos, como el conteo por cámara de Neubauer, se demostró el favorable crecimiento celular de la microalga, en medio de cultivo Foliagro con glicerol, como fuente de carbono adicional.

Del mismo modo se confirmó por medios cualitativos, como el cambio de coloración (a verde oscuro), y la disminución de volumen inicial, la clara adaptación de la micro-alga al medio de cultivo.

Se determinó que las concentraciones de glicerol en medio Foliagro, que mayor cantidad de biomasa micro-algar produjeron, fueron $15 \%$,
$10 \%$ y $20 \%$ respectivamente. Se presentaron también los menores tiempos de adaptación al medio de cultivo. La micro-alga presentó un crecimiento directamente proporcional al aumento de la concentración de glicerol, hasta un $15 \%$. A partir de allí, se presentó una saturación de la fuente de carbono en el medio de cultivo, provocando una aparente inhibición en el crecimiento celular. Se reportó un crecimiento máximo de $2,29 \times 10^{7} \mathrm{Cel} / \mathrm{ml}$ para la concentración de $15 \%$ de glicerol.

El perfil lipídico obtenido, a partir de la biomasa micro-algar, tiene un alto contenido de lípidos poliinsaturados (PUFAs), equivalente al $46,1 \%$. Un $29.5 \%$ de ácidos saturados y $24.4 \%$ de ácidos mono-insaturados.

Considerando la valoración económica que podría darse al glicerol, al escalar a nivel de planta piloto y posteriormente para la industria alimenticia, el método llevado a cabo para la extracción de omegas, podria viabilizarse económicamente el desarrollo de nuevas tecnologías, pudiendo aprovechar los lípidos en la producción de biodiesel, ambientalmente sostenible.

\section{REFERENCIAS}

Abalde, J. (1995) "Concepción, Micro-algas: Cultivo y Aplicaciones". Coruña: Universidad de la Coruña p. 21

Ángel, C., Pimenta, W. (2012) "Evaluación de la incidencia del nitrógeno en el medio de cultivo bold basal para la producción de biomasa de la micro-alga chlorella vulgaris en un foto-biorreactor, para la obtención de ácidos grasos". Tesis de investigación Ingeniería Química. Universidad de América. Facultad de Ingeniería, Programa de Ingeniería Química. p. 64.
Amaro, H., Guedes, A., Catarina. \& Malcata, F. (2011) "Advances and perspectives in using micro-algae to produce biodiesel". En: Applied Energy. Vol., 88, No. 10. p. 3406.

Anónimo. Secado por aspersión. [En línea].Universidad Nacional de Colombia, Sede Manizales. [Citado el

27 de noviembre de 2012]. Disponible desde Internet en: <http://www.virtual.unal. edu.co/cursos/sedes/manizales/4070035/lecciones/cap7/ leccion7_7.htm>
Arias, M. et al. (2013) "Producción de biodiesel a partir de micro-algas: parámetros del cultivo que afectan la producción de lípidos". Acta biol. Colomb. Vol. $18 \mathrm{~N}^{\circ}$. 1. p. 56

Bahadar, A. \& Bilal M. (2013) "Progress in energy from micro-algae: A review, Renewable and sustainable energy reviews". V. 27. p. 148 rón, J.

Belén, C., Silva, E. \& Ba-

(2013) "Aplicaciones biotecnológicas de micro-algas: caso de estudio. Proyecto de Producción de Micro-algas 
para obtención de Biocombustibles". Segundas Jornadas de Biotecnología Universidad Nacional de Cuyo, Argentina.

Benavente, J. \& Montañez, J., N Aguilar., A Mendez., B Valdivia. Tecnología de Cultivo de Micro-algas en Foto-biorreactores."(2012). Acta Química Mexicana enero-junio 2012. Año 4 No 7.

Bligh, E. \& Dyer, W. (1959) "A rapid method for total lipid extraction and purification". Canadian Journal of Biochemistry and Physiology, 37(8). p. 911-917.

Camacho et al. "Cultivo y elaboración de un producto comestible de Chlorella vulgaris". En: UNAM. Recuperado de: <http://www.feriadelasciencias. unam.mx/anteriores/xviiferia/21.pdf>, p. 5.

Clavell, L., Pedrique, M. (1992). "Microbiología. Manual de Métodos Generales" (segunda edición). Facultad de Farmacia. Universidad Central de Venezuela.

Da Silva, G., Mack, M. \& Contiero, J. (2009) "Glycerol: A promising and abundant carbon source for industrial microbiology. Biotechnology Advances. p. 30-39.Disponible en: http:// www.ecopetrol.com.co/especiales/Revistalnnova7ed/idi04. html. Revisado Junio de 2014

Demirbas. A., Demirbas, F. (2011) "Importance of microalgae as a source of biodiesel".
Energy Conversion and Management Vol 52 (1).

Deschenes, J., Boudreau, A., Rejean, T. (2015) "Mixotrophic production of microalgae in pilot-scale photobioreactors: Practicability and process considerations". Algal Research 10: 80-86.

Estevez-Landezabal,L., Barajas,A., Barajas, C, Kafarov, V.(2013) "Improvement of lipid productivity on Chlorella vulgaris using waste glycerlol and sodium acetate". Ciencia Tecnologia y Futuro Vol 5 (2):113-126

Ferrero, I. (2011) "Producción de Biodiesel a partir de Micro-algas como alternativa a los cultivos clásicos". Universidad Nacional del Litoral. Bioquímica y Ciencias Biológicas. p. 5.

García, J., Molina, E., García, F., Sánchez P., \& Giménez, A. (1993) "Cuantificación de ácidos grasos a partir de biomasa micro-algar". En Grasas y Aceites Departamento de Ingeniería Química. Facultad de Ciencias Experimentales. Universidad de Almeria.

Garibay, A. et al. (2009) "Biodiesel a Partir de Micro-algas". Instituto de Biotecnología, Universidad Nacional Autónoma de México. BioTecnología, Año, Vol. 13 No. 3, p. 42.

Gary., A., (2009) "A review of lab scale photobioreactor development". Proceedings of the South Academy of Science, Vol. 88. p. 34.

González, A., Kafarov, V., Guzmán, A. (2010) "Desarrollo de métodos de extracción de aceite en la cadena de producción de biodiesel a partir de micro-algas". En: Prospect. Diciembre. Vol. 7. no. 2. p. 54.

Instituto Colombiano de Normas Técnicas y Certificación. (2008) "Documentación. Presentación de tesis, trabajos de grado y otros trabajos de investigación". NTC 1486. Sexta actualización. Bogotá: El Instituto. p.1.

cias bibliográficas, contenido, forma y estructura.. NTC 5613. Bogotá: El Instituto, 2008, p.1- 2 _. Referencias documentales para fuentes de información electrónica. NTC 4490. Bogotá: El Instituto, 1998, p.2

Jaramillo, J. (2011) "Evaluación tecno-económica de la producción de biocombustibles a partir de micro-algas". Trabajo de grado Magister en Ingeniería Química. Manizales, Colombia. Universidad Nacional de Colombia. Facultad de Ingeniería y Arquitectura, Maestría en Ingeniería-Ingeniería Química. p. 24.

Jarunan, P., Piyawan, S. \& Thidarat, N. (2005) "Fatty acids composition of 10 micro-algar species". Songklanakarin J. Sci. Technol. 27(6). p. 1179-1187 
LIINEA DE INVESTIGACIÓN: APROVECHAMIENTO DE RESIDUOS AGROINDUSTRIALES

Kong, W., Song, H., Cao, Y., Yang, H., Hua, S. \& Xia, C. (2011). "The characteristics of biomass production, lipid accumulation and chlorophyll biosynthesis of Chlorella vulgaris under mixotrophic cultivation". African journal of Biotechnology. 10(55). p. 11620-11630.

Li,X., Xu,H., Wu,Q. (2007) "Large - Scale Biodiesel production from microalgae chlorella protothecoides through heterotrophic cultivation in bioreactors". Biotechnology and Bioengineering Vol 98: 764-771.

Loaiza, F. (2003) "Cinética de la reacción de transesterificación del aceite de higuerilla en la obtención de biodiesel". Universidad Nacional de Colombia. p. 26

Madigan, M., Martinlo, J., Parker. (2010) "Brock biology of microorganisms" Ninth Edition. Ed Prentice Hall. ISBN 0-13-081922-0

Monthieu, C. (2010) "Estudio técnico económico de la extracción de los lípidos de las micro-algas para la producción de biodiesel". Universidad pontificia comillas, escuela técnica superior de ingeniería (ICAI). Madrid, Junio. p. 70.

Morales, W, N Polish, A Sequeira, E Chamorro, E Herrero (2010) "Purificación de glicerol obtenido a partir de síntesis de biodiesel de aceite crudo de algodón". Avances en Energías Renovables y Medio Ambiente vol 14.
Moser, B. (2009) "Biodiesel production, properties, and feedstocks". In Vitro Cellular \& Developmental Biology - Plant, 45(3). p. 229-266.

Nag, D., et al. (2012). "Recent Trends on the Development of Photobiological Processes and Photobioreactors for the Improvement of Hydrogen Production". En: International Journal of Hydrogen Energy. Vol., 35. No 19 (Oct). p. 10228.

O'grady, J. \& Morgan, J. (2011) "Heterotrophic growth and lipid production of Chlorella protothecoides on glycerol". Bioprocess Biosyst Eng. 34:121-125

Olguín, L. (2004) "Cromatografía de Gases. Métodos en Biotecnología". Universidad Nacional Autónoma de México. Junio. p. 1.

Ortiz, E. \& Castro, K. (2013) "Determinación de la incidencia de la relación carbono: nitrógeno en la producción de biomasa y ácidos grasos en la microalga chlorella vulgaris a escala de laboratorio". Tesis de investigación Ingeniería Química. Universidad de América. Facultad de Ingeniería, Programa de Ingeniería Química. p. 51.

Pestana, R,M., Pestana R. (2012) "Producción de micro-algas UTEX 1803 usando como sustrato glicerol residual a escala de laboratorio". Tesis de Grado de Ingeniero Quími- co. Universidad Industrial de Santander. p. 11.

Portilla, M., Eraso, S., Galé, C., Garcia, I., Moler, J. \& Blanca, M. (2006) "Manual práctico del paquete estadístico SPSS para Windows" ( $3^{\mathrm{a}}$ edición revisada). Universidad Pública de Navarra: Navarra.

Posada, J., Cardona, C. (2010) "Análisis de la refinación de glicerina obtenida como coproducto en la producción de biodiesel". Ingenieria y Universidad vol 14 No 1

Rodríguez, A., Serrano, L. \& Firson, A. (2012) "Efecto de la relación Carbono/ Nitrógeno en la productividad de Biomasa y Lípidos en cultivos de Chlorella Vulgaris Utex 1803 en Foto-biorreactores a escala de laboratorio". Universidad Industrial de Santander. Ingeniería Química. P. 15.

Salazar, L. (2012) "Evaluación de métodos de extracción de aceite de micro-algas para la producción de biodiesel". Proyecto de grado. Facultad de ingeniería. Universidad de Piura. p. 46.

Serrano, L. (2012). "Estudio de cuatro cepas nativas de micro-algas para evaluar su potencial uso en la producción de biodiesel" Tesis de investigación Magister en Ingeniería Química. Universidad Nacional de Colombia. Facultad de Ingeniería, Departamento de Ingeniería Química y Ambiental. p. 41. 
Slade, R., \& Bauen, A. (2013) "Micro-algae cultivation for biofuels: Cost, energy balance, environmenta impacts and future prospects". Biomass and Bioenergy. V. 53. p. 29.

Solovchenko, A. (2011) "Physiological Role of Neutral Lipid Accumulation in Eukaryotic Micro-algae under Stresses"; Faculty of Biology, Moscow State University; Moscow.

Torres, C., Rios,S., Torras.,C. (2013) "Microalgaebased biodiesel: A multicriteria analysis of the production process". Bioresource Technology Vol 147: 7-16

Valencia, M. \& Cardona, C. (2013) "Evaluación ambiental para procesos que usan residuos de la industria de los biocombustibles como materias primas". p. 106.
Wang, C., LI, H., Wang, Q., WEI, P. (2010) "Effect of pH on growth and lipid content of Chlorella vulgaris cultured in biogas slurry". En: Sheng Wu Gong Cheng Xue Bao. Vol., 26, No. 8 (Ago); p. 1074.

Kong, W. et al (2013) "Chlorella vulgaris Cultivation on Glycerol and Glucose" Food Teehnol Biotechnol. 51 (1). p. 62-69.

Disponible en: http:/l www.faciasuncoma.com.ar/ Carreras/Saneamiento $\% 20$ Ambiental/MicrobiologiaAmbiental/8\%20RECUENTO.pdf. Revisado Julio de 2014

Xiufeng, L., Han, X., Qingyu, Wu. (2007) "Large-Scale Biodiesel Production From Micro-alga Chlorella protothecoides Through Heterotrophic Cultivation in Bioreactors". De- partment of Biological Sciences and Biotechnology, Tsinghua University, Beijing. Wiley InterScience. Biotechnology and Bioengineering, Vol. 98, No 4, November 1.

Xu,H., Miao,X., Wu,Q. (2006). High quality biodiesel production from a microalga Chlorella prototecoides by heterotophic growth in fermenters. Journal of Biotechnology 126: 499-507.

Yun, Y., Lee S., Park, J., Lee, C. \& Yang, J. (1997) "Carbon dioxide fixation by algar cultivation using waste water nutrients". J. of Chem. Technol. Biotechnol. 